\title{
Prognostic nomogram for nonresectable pancreatic cancer treated with gemcitabine-based chemotherapy
}

\begin{abstract}
T Hamada ${ }^{1}$, Y Nakai ${ }^{1}$, H Yasunaga ${ }^{2}$, H Isayama ${ }^{*}, 1$, H Matsui ${ }^{2}$, N Takahara ${ }^{3}$, T Sasaki ${ }^{1}$, K Takagi ${ }^{1}$, T Watanabe ${ }^{3}$, H Yagioka ${ }^{4}$, H Kogure ${ }^{1}$, T Arizumi ${ }^{5}$, N Yamamoto ${ }^{1}$, Y Ito $^{6}$, K Hirano ${ }^{1}$, T Tsujino ${ }^{6}$, M Tada ${ }^{1}$ and K Koike ${ }^{1}$

${ }^{1}$ Department of Gastroenterology, Graduate School of Medicine, The University of Tokyo, 7-3-1 Hongo, Bunkyo-ku, Tokyo 113-8655, Japan; ${ }^{2}$ Department of Health Economics and Epidemiology Research, School of Public Health, The University of Tokyo, 7-3-1 Hongo, Bunkyo-ku, Tokyo 113-8655, Japan; ${ }^{3}$ Department of Gastroenterology, Kanto Central Hospital, 6-25-1 Kami-Yoga, Setagaya-ku, Tokyo 158-8531, Japan; ${ }^{4}$ Department of Gastroenterology, JR Tokyo General Hospital, 2-1-3 Yoyogi, Shibuya-ku, Tokyo 151-8528, Japan; ${ }^{5}$ Department of Gastroenterology, Mitsui Memorial Hospital, 1 Izumi-cho, Kanda, Chiyoda-ku, Tokyo 101-8643, Japan and 'Department of Gastroenterology, Japanese Red Cross Medical Center, Tokyo, 4-1-22 Hiroo, Shibuya-ku, Tokyo 150-8935, Japan
\end{abstract}

Background: A nomogram is progressively being used as a useful predictive tool for cancer prognosis. A nomogram to predict survival in nonresectable pancreatic cancer treated with chemotherapy has not been reported.

Methods: Using prospectively collected data on patients with nonresectable pancreatic cancer receiving gemcitabine-based chemotherapy at five Japanese hospitals, we derived a predictive nomogram and internally validated it using a concordance index and calibration plots.

Results: In total, 531 patients were included between June 2001 and February 2013. The American Joint Committee on Cancer (AJCC) TNM stages were III and IV in 204 and 327 patients, respectively. The median survival time of the total cohort was 11.3 months. A nomogram was generated to predict survival probabilities at 6, 12, and 18 months and median survival time, based on the following six variables: age; sex; performance status; tumour size; regional lymph node metastasis; and distant metastasis. The concordance index of the present nomogram was higher than that of the AJCC TNM staging system at 12 months (0.686 vs 0.612$)$. The calibration plots demonstrated good fitness of the nomogram for survival prediction.

Conclusions: The present nomogram can provide valuable information for tailored decision-making early after the diagnosis of nonresectable pancreatic cancer.

Pancreatic cancer is the fourth leading cause of cancer death in the United States (Siegel et al, 2013) and the fifth in Japan. It is often nonresectable at the time of diagnosis, and is generally associated with a poor prognosis. However, recent advances in chemotherapy have prolonged the survival time of patients with nonresectable pancreatic cancer (Nakai et al, 2010; Conroy et al, 2011; Sun et al, 2012). Systemic administration of gemcitabine has been the mainstream first-line chemotherapy for nonresectable pancreatic cancer, since Burris et al (1997) demonstrated the superiority of gemcitabine over 5-fluorouracil. More recently, gemcitabine-based combination chemotherapies have been intensely investigated (Heinemann et al, 2006; Nakai et al, 2012b; Ueno et al, 2013), but few trials have shown their superiority over gemcitabine monotherapy (Moore et al, 2007; Von Hoff et al, 2013).

In this setting, reliable prognostic information is desired for tailored management of individual patients with advanced

*Correspondence: Professor H Isayama; E-mail: isayama-tky@umin.ac.jp

Received 4 December 2013; revised 14 February 2014; accepted 20 February 2014; published online 18 March 2014

(c) 2014 Cancer Research UK. All rights reserved 0007-0920/14 
pancreatic cancer receiving gemcitabine-based chemotherapy. The most widely used staging system for pancreatic cancer is the American Joint Committee on Cancer (AJCC) TNM staging system (Edge et al, 2010). However, it is relatively nondiscriminatory for survival prediction in nonresectable pancreatic cancer, which is mostly diagnosed as TNM stage III or IV.

A nomogram is a simple graphical presentation of a multivariate predictive model showing the impact of each included variable on an outcome of interest that provides a numerical probability of the outcome (Iasonos et al, 2008), and is progressively being used as a useful predictive tool for cancer prognosis in the field of oncology (Kattan et al, 2002, 2003; International Bladder Cancer Nomogram Consortium et al, 2006; Touijer and Scardino, 2009). Several prognostic factors for survival of nonresectable pancreatic cancer have been reported (Ishii et al, 1996; Ueno et al, 2000; Sezgin et al, 2005; Nakai et al, 2008, 2011), but were evaluated separately in different cohorts. One of the strengths of a nomogram is the ability to integrate multiple prognostic factors into a single numerical estimate of survival in an individual patient and thus provide an individualised prediction of survival. A nomogram to predict survival after resection of pancreatic cancer was generated by Brennan et al (2004) and has been validated externally as well as internally (Ferrone et al, 2005). However, to our knowledge, a nomogram for survival prediction in nonresectable pancreatic cancer treated with chemotherapy has not been reported.

The aim of this study was to generate and internally validate a nomogram to predict survival in patients with nonresectable pancreatic cancer receiving gemcitabine-based chemotherapy.

\section{MATERIALS AND METHODS}

Patients. From a collaborative prospective database of patients with pancreatic cancer including data from the University of Tokyo Hospital and affiliated hospitals, we identified consecutive patients who were diagnosed with nonresectable pancreatic cancer and subsequently received gemcitabine-based chemotherapy as the first-line anticancer treatment between June 2001 and February 2013. Pancreatic cancer was diagnosed by pathological examination or typical radiographic findings or by a clinical follow-up of at least 6 months. Nonresectability was confirmed via consultation with the departments of surgery and anaesthesiology in each hospital. Patients were followed-up at least every 2 weeks on an outpatient basis, and the tumour responses were evaluated by computed tomography, which was performed at baseline and then after every two cycles (8 weeks), according to the Response Evaluation Criteria in Solid Tumors, version 1.0 (Therasse et al, 2000). Follow-up was performed until October 2013. The study was approved by the ethics committee at each institution.

Gemcitabine-based chemotherapy. Patients received gemcitabine alone or in combination with S-1, candesartan, or erlotinib. The regimen of each chemotherapy was as follows. For gemcitabine monotherapy, gemcitabine was administered intravenously at $1000 \mathrm{mg} \mathrm{m}^{-2}$ on days 1,8 , and 15 within each 4 -week cycle. For gemcitabine and S-1, gemcitabine was administered intravenously at $1000 \mathrm{mg} \mathrm{m}^{-2}$ on days 1 and 15 , and S-1 was given orally b.i.d. from days 1 to 14 within each 4 -week cycle. The doses of S-1 were determined according to the body surface area (BSA) as follows: $\mathrm{BSA} \leqslant 1.25 \mathrm{~m}^{2}, 80 \mathrm{mg}$ per day; $1.25 \mathrm{~m}^{2}<\mathrm{BSA} \leqslant 1.5 \mathrm{~m}^{2}, 100 \mathrm{mg}$ per day; and $B S A \geqslant 1.5 \mathrm{~m}^{2}, 120 \mathrm{mg}$ per day. For gemcitabine and candesartan, gemcitabine monotherapy plus oral candesartan at a dose of 4-32 mg per day was administered within each 4-week cycle. For gemcitabine and erlotinib, gemcitabine monotherapy plus oral erlotinib at a dose of 100 or $150 \mathrm{mg}$ per day was administered. Some of the patients were enrolled in our clinical trials: GEMSAP (Nakai et al, 2012b) and GECA1/2 (Nakai et al, 2012a, 2013).

AJCC TNM staging system. In the current staging system utilising T, N, and $\mathrm{M}$ factors (AJCC Cancer Staging Manual, 7th Edition, 2010) (Edge et al, 2010), the pancreatic cancer stages are categorized as follows: Stage IA, T1 N0 M0; Stage IB, T2 N0 M0; Stage IIA, T3 N0 M0; Stage IIB, T1-3 N1 M0; Stage III, T4 N-any M0; and Stage IV, T-any N-any M1.

Statistical analysis. Survival time was defined as the time from initiation of chemotherapy to all-cause death. Patients who were alive at the last follow-up or lost to follow-up were treated as censored at the time of last follow-up. Survival times were estimated using the Kaplan-Meier method and compared using the log-rank test. The $95 \%$ confidence interval (CI) of the median survival time (MST) was calculated (Brookmeyer and Crowley, 1982) and that of the survival rate was also calculated by the formula of Greenwood (Kalbfleisch and Prentice, 1980).

The multivariate Cox proportional hazards model was used to generate a nomogram to predict survival probabilities at 6,12 , and 18 months and MST. The following variables were included: age; sex; performance status based on the criteria of the Eastern Cooperative Oncology Group; tumour size; regional lymph node metastasis (AJCC N-factor); and distant metastasis (AJCC $\mathrm{M}$-factor). Tumour size was defined as the maximum diameter of a primary tumour based on the findings of computed tomography. The performance status values of 2 and 3 were grouped because of the small numbers of patients. The proportional hazards assumption of each variable was verified by Schoenfeld residual plots.

The newly generated nomogram was internally validated via two steps. First, the nomogram was subjected to bootstrapping with 1000 resamples to calculate a relatively unbiased measure of its ability to discriminate the survival times of two patients(concordance index).The concordance index quantifies the level of concordance between predicted probabilities and actual outcomes, and ranges from 0.5 (no discrimination at all) to 1.0 (perfect discrimination). In other words, it reflects the probability that a patient with a lower probability of survival predicted via the nomogram dies earlier than another patient with a higher predicted probability, when considering two patients randomly selected from the study population. Second, the predicted probability was compared with the observed frequency in the total study population, again using bootstrapping with 1000 resamples to reduce an overfit bias (calibration).

The superiority of the present nomogram over the AJCC TNM staging system for survival prediction was confirmed as follows. The bias-corrected concordance indexes of the nomogram and the AJCC TNM staging system were calculated to compare their predictive abilities for survival. The heterogeneity of survival within each AJCC stage was evaluated by demonstrating histograms of nomogram-predicted survival probabilities. Stratification of actual survival via the nomogram-predicted probabilities was illustrated by categorising the total cohort according to quartiles of the probabilities and comparing survival times between the groups.

All analyses were performed using $\mathrm{R}$ software version 2.15 .2 (R Development Core Team; http://www.r-project.org) and the RMS package developed by Harrell (Harrell et al). Values of $P<0.05$ were considered statistically significant, and all tests were two sided.

\section{RESULTS}

Patient characteristics. In total, 531 consecutive patients who received gemcitabine-based chemotherapy as first-line 
chemotherapy for nonresectable pancreatic cancer were identified. The patient characteristics are summarised in Table 1. The MST of the study population was 11.3 months (95\% CI: $10.2-12.8$ months), and the survival rates at 6,12 , and 18 months were $73.5 \%$ (95\% CI: 69.8-77.5\%), 47.2\% (95\% CI: 42.9-51.9\%), and 28.9\% (95\% CI: 24.9-33.5\%), respectively. At last follow-up, 442 patients $(83.2 \%)$ had died, with a median follow-up time of 9.4 months.

Generation and internal validation of a prognostic nomogram. A nomogram was generated via the Cox proportional hazards model including the above mentioned variables, and is demonstrated with brief instructions for its usage in Figure 1. The results of the underlying univariate and multivariate Cox models for survival time are shown in Table 2 . The nomogram predicts the survival probabilities of 6,12 , and 18 months and the MST after initiation of chemotherapy in a given patient. For example, a 65year-old ('Points' $=10$ ) male ('Points'=5) patient with performance status of 1 ('Points'=20), tumour size of $60 \mathrm{~mm}$ ('Points' = 33), regional lymph node metastasis ('Points' $=15$ ), and absence of distant metastasis ('Points' $=0$ ) has a 'Total Points' score of 69, which corresponds to 6-, 12-, and 18-month-predicted survival probabilities of $80 \%, 54 \%$, and $34 \%$, respectively, and to predicted MST of 13 months.

Table 1. Characteristics of the 531 patients with nonresectable pancreatic cancer who received gemcitabine-based chemotherapy

\begin{tabular}{|c|c|}
\hline \multicolumn{2}{|l|}{ Age, years } \\
\hline Median (range) & $68(35-89)$ \\
\hline \multicolumn{2}{|l|}{ Sex, $n(\%)$} \\
\hline Male/female & $306 / 225(57.6 / 42.4)$ \\
\hline \multicolumn{2}{|l|}{ ECOG performance status, $n(\%)$} \\
\hline $0 / 1 / 2 / 3$ & $190 / 276 / 63 / 2(35.8 / 51.9 / 11.9 / 0.4)$ \\
\hline \multicolumn{2}{|c|}{ Location of primary tumour, $n(\%)$} \\
\hline Head/body/tail of the pancreas & 267/157/107 (50.2/29.6/20.2) \\
\hline \multicolumn{2}{|l|}{ Tumour size, (mm) } \\
\hline $\begin{array}{l}\text { Median (range) } \\
\text { Distant metastasis, } n(\%)\end{array}$ & $\begin{array}{c}35(14-170) \\
327(61.6)\end{array}$ \\
\hline \multicolumn{2}{|l|}{ Site of metastasis, $n(\%)$} \\
\hline Liver/lung/lymph node/peritoneum & $220 / 60 / 271 / 84(41.4 / 11.3 / 51.0 / 15.8)$ \\
\hline \multicolumn{2}{|l|}{ CEA, IU/I } \\
\hline Median (range) & $5.2(0.7-3948)$ \\
\hline \multicolumn{2}{|l|}{ CA19-9, IU/I } \\
\hline Median (range) & $493(1-574000)$ \\
\hline \multicolumn{2}{|l|}{ AJCC stage, $n(\%)$} \\
\hline III/IV & $204 / 327(38.4 / 61.6)$ \\
\hline \multicolumn{2}{|l|}{ Chemotherapy, n (\%) } \\
\hline $\begin{array}{l}\text { Gemcitabine monotherapy } \\
\text { Gemcitabine and S-1 } \\
\text { Gemcitabine and candesartan } \\
\text { Gemcitabine and erlotinib }\end{array}$ & $\begin{array}{c}374(70.5) \\
108(20.3) \\
41(7.7) \\
8(1.5)\end{array}$ \\
\hline $\begin{array}{l}\text { Abbreviations: } A J C C=\text { American Joint Comr } \\
\text { 19-9; } C E A=\text { carcinoembryonic antigen; } E C\end{array}$ & $\begin{array}{l}\text { ttee on Cancer; CA 19-9 = carbohydrate antigen } \\
\mathrm{G}=\text { Eastern Cooperative Oncology Group. }\end{array}$ \\
\hline
\end{tabular}

The bias-corrected concordance indexes of the present nomogram were higher than those of the AJCC TNM staging system at all time points ( 0.686 vs 0.612 for 6-month survival; 0.686 vs 0.612 for 12-month survival; and 0.686 vs 0.611 for 18-month survival).These results demonstrated that the discrimination via the newly generated nomogram was superior to the grouping via the AJCC TNM staging system. Calibration plots of the 6-, 12-, and 18-month survival are shown in Figure 2. The mean absolute errors between the observed and predicted probabilities were $0.019,0.045$, and 0.038 for 6-, 12-, and 18-month survival, respectively, and the errors for $90 \%$ of the study population were within $0.046,0.027$, and 0.057 , respectively.

AJCC TNM staging and nomogram-predicted survival probabilities. Figure 3 illustrates histograms of nomogrampredicted survival probabilities at 12 months after initiation of chemotherapy within each of the TNM stages (III and IV). There was considerable heterogeneity in the nomogram-predicted survival probabilities even for the same TNM stage.

Survival time according to risk stratification based on nomogram-predicted survival probabilities. On the basis of nomogram-predicted survival probabilities, the patients were categorised into the following quartiles of risk: very low ('Total Points': 554$)$; low (55-81); high (82-105); and very high $(\geqslant 106)$. Figure 4 illustrates the Kaplan-Meier survival curves according to the nomogram-based groupings. The survival times were significantly differentiated between the groups $(P<0.001)$. The MSTs in the very low-, low-, high-, and very high-risk groups were 17.5 (95\% CI: 15.4-22.9), 13.7 (95\% CI: 11.6-16.0), 8.9 (95\% CI: 7.9-10.4), and 5.5 (95\% CI: 4.7-7.5) months, respectively.

\section{DISCUSSION}

The present prognostic nomogram derived from prospectively collected data on 531 patients from five hospitals was shown to provide improved ability for individualised survival prediction in patients with nonresectable pancreatic cancer receiving gemcitabine-based chemotherapy, compared with the existing TNM staging system. By using this nomogram, individualisation of patient counselling and decision-making regarding management can be promoted.

Patients with nonresectable pancreatic cancer have a very high probability of ultimately dying of their primary disease. However, given the improved survival via recent advances in chemotherapy for this condition (Nakai et al, 2010; Conroy et al, 2011; Sun et al, 2012), the importance of tailored management of patients with nonresectable pancreatic cancer has increased, and clinical physicians and patients alike desire reliable prognostic information tailored to individual patients. Although the AJCC has developed and revised a TNM staging system for pancreatic neoplasms (Edge et al, 2010), it was not specifically developed for survival prediction of nonresectable cases, but instead was rather related to resectability evaluation and preoperative staging. In this staging system, most patients with nonresectable pancreatic cancer are diagnosed as stage III or IV, and are thus only dichotomised. Therefore, the TNM staging system is relatively nondiscriminatory as a means for survival prediction of nonresectable pancreatic cancer treated with chemotherapy. Actually, within the group at each TNM stage in the present study, considerable heterogeneity was found in terms of nomogram-predicted survival probabilities among the patients (Figure 3). In other words, the patients were associated with various survival times, even if they were diagnosed as the same TNM stage.

A nomogram was developed as a statistical tool to provide the overall probability of a specific outcome via a simple graphical 


$\begin{aligned} & \text { Points } \\ & \text { Age (years) }\end{aligned}$
$\begin{aligned} & \text { Performance status } \\ & \text { Tumour size (mm) }\end{aligned}$
Regional lymph node metastasis
Distant metastasis
Total points

Figure 1. A nomogram to predict the survival of patients with nonresectable pancreatic cancer receiving gemcitabine-based chemotherapy. To estimate survival in a given patient, the 'Total Points' score is calculated by summing the respective 'Points'values corresponding to each variable. Using this 'Total Points' score, the survival probabilities at 6, 12, and 18 months and the median survival time can be predicted according to the lower scales.

Table 2. Univariate and multivariate Cox proportional hazards models to predict survival in patients with nonresectable pancreatic cancer receiving gemcitabine-based chemotherapy

\begin{tabular}{|c|c|c|c|c|c|c|}
\hline & \multicolumn{3}{|c|}{ Univariate analysis } & \multicolumn{3}{|c|}{ Multivariate analysis } \\
\hline & HR & $95 \% \mathrm{Cl}$ & $P$-value & HR & $95 \% \mathrm{Cl}$ & $P$-value \\
\hline Age & 0.989 & $0.979-0.998$ & 0.024 & 0.993 & $0.983-1.004$ & 0.202 \\
\hline \multicolumn{7}{|l|}{ Sex } \\
\hline $\begin{array}{l}\text { Female } \\
\text { Male }\end{array}$ & $\begin{array}{c}\text { Reference } \\
1.074\end{array}$ & $0.885-1.306$ & 0.473 & $\begin{array}{c}\text { Reference } \\
1.086\end{array}$ & $0.892-1.323$ & 0.413 \\
\hline \multicolumn{7}{|c|}{ Performance status } \\
\hline $\begin{array}{l}0 \\
1 \\
2-3\end{array}$ & $\begin{array}{c}\text { Reference } \\
1.413 \\
4.359\end{array}$ & $\begin{array}{l}1.148-1.742 \\
3.079-6.067\end{array}$ & $\begin{array}{r}0.001 \\
<0.001\end{array}$ & $\begin{array}{c}\text { Reference } \\
1.393 \\
4.706\end{array}$ & $\begin{array}{l}1.131-1.720 \\
3.292-6.622\end{array}$ & $\begin{array}{r}0.002 \\
<0.001\end{array}$ \\
\hline Tumour size & 1.015 & $1.010-1.021$ & $<0.001$ & 1.009 & $1.004-1.014$ & 0.001 \\
\hline \multicolumn{7}{|c|}{ Regional lymph node metastasis } \\
\hline $\begin{array}{l}\text { No } \\
\text { Yes }\end{array}$ & $\begin{array}{c}\text { Reference } \\
1.551\end{array}$ & $1.279-1.882$ & $<0.001$ & $\begin{array}{c}\text { Reference } \\
1.276\end{array}$ & $1.043-1.562$ & 0.018 \\
\hline \multicolumn{7}{|c|}{ Distant metastasis } \\
\hline $\begin{array}{l}\text { No } \\
\text { Yes }\end{array}$ & $\begin{array}{c}\text { Reference } \\
2.098\end{array}$ & $1.713-2.580$ & $<0.001$ & $\begin{array}{c}\text { Reference } \\
1.866\end{array}$ & $1.514-2.308$ & $<0.001$ \\
\hline
\end{tabular}

presentation, and was shown to be more accurate than conventional staging systems for predicting prognosis in various malignancies (Kattan et al, 2002, 2003; International Bladder Cancer Nomogram Consortium et al, 2006) and benign diseases (Klein et al, 2002; Sugihara et al, 2013a, b). As a nomogram is simple and understandable, it has been easily introduced into daily clinical practice. In addition, a nomogram can generate individualised predictions, and thus patients can be evaluated for their participation in clinical trials using this tool. For example, several randomised controlled trials have included expected survival time in their eligibility criteria, and a nomogram that can more accurately predict survival can provide valuable information for this consideration, potentially making such trials more sophisticated (Iasonos et al, 2008). 

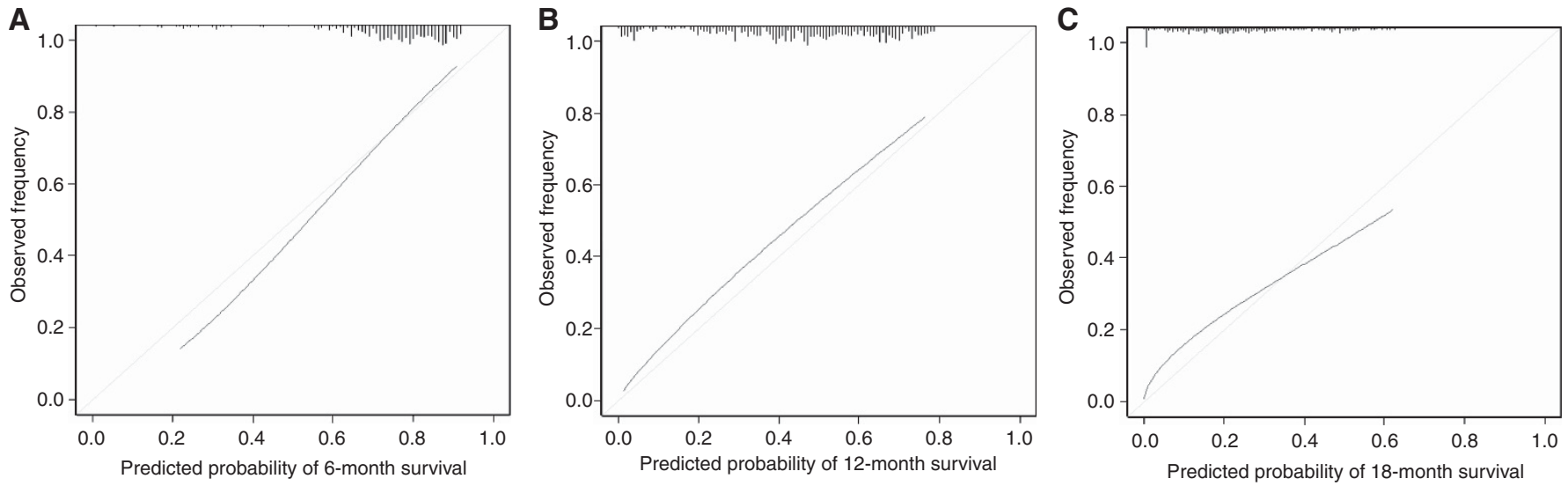

Figure 2. Calibration plots of the present nomogram for 6-, 12-, and 18-month survival that were adjusted by bootstrapping with 1000 resamples ((A) 6 months; (B) 12 months; and (C) 18 months). The diagonal line indicates the ideal nomogram reference.

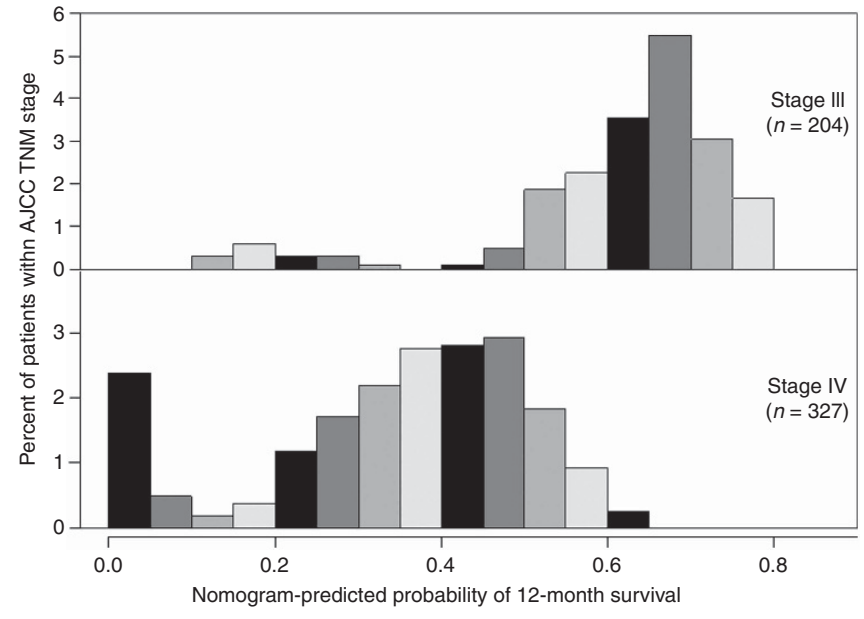

Figure 3. Histograms of nomogram-predicted survival probabilities at 12 months after initiation of chemotherapy within each AJCC TNM stage. Abbreviation: AJCC, American Joint Committee on Cancer.

In this study, we aimed to generate and internally validate a nomogram to overcome the above mentioned drawbacks of the TNM staging system as a means for survival prediction in patients with nonresectable pancreatic cancer receiving chemotherapy. The present nomogram included the following six variables that can be readily determined, thus providing information on predicted survival at the time of chemotherapy initiation: age; sex; performance status; tumour size; regional lymph node metastasis; and distant metastasis. Age and sex are baseline characteristics of patients. Performance status, which was reported to be a significant risk factor for survival in nonresectable pancreatic cancer (Ishii et al, 1996; Ueno et al, 2000; Sezgin et al, 2005), can be determined by physical examinations, and the remaining factors by computed tomography, which is routinely performed for patients with unresectable pancreatic cancer. In addition, no specific molecular markers (Liu et al, 2012; Lee et al, 2013) are required for this nomogram. Therefore, survival prediction via the present nomogram can be made immediately at an outpatient clinic without any additional costs, potentially providing valuable information for decision-making regarding treatments early after diagnosis of the disease. Carbohydrate antigen 19-9 (CA 19-9) is widely recognised as a prognostic factor for pancreatic cancer (Ueno et al, 2000; Ikeda et al, 2001; Nakai et al, 2008). However, patients whose red blood cell phenotyping for both Lewis A and B antigens is negative are unable to secrete CA 19-9 into their serum. Therefore, we did not include this variable in the model to secure usability for the general

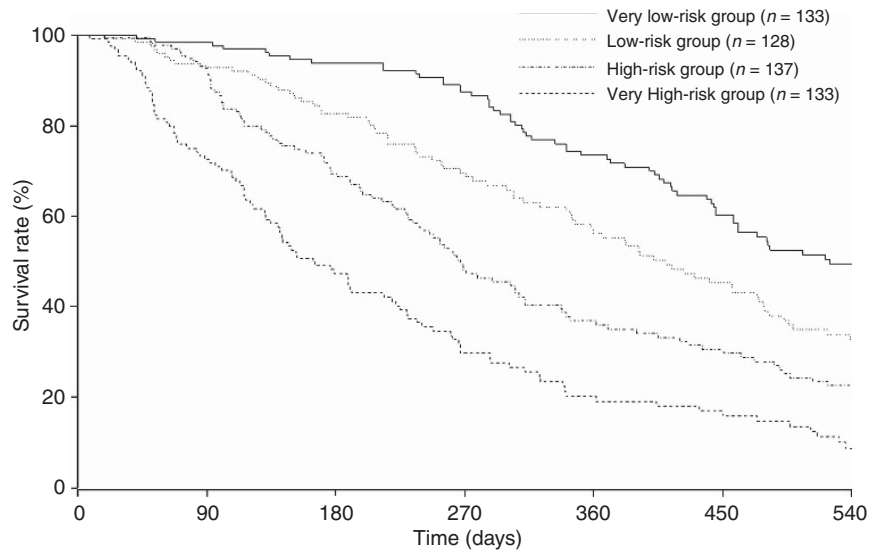

Figure 4. Kaplan-Meier survival curves according to the quartiles of nomogram-predicted survival probabilities. The survival times were significantly differentiated between the groups $(P<0.001)$.

population, but did include tumour size, which is positively correlated with CA 19-9 (Sakahara et al, 1986; Tian et al, 1992).

Overall, the internal validation demonstrated good fitness for survival prediction at several specific time points, as the predicted survival probabilities at 6,12 , and 18 months estimated via the nomogram were closely aligned with the actual survival times. Again, Figure 3 emphasizes that our nomogram provides a more differentiating prediction model compared with the AJCC TNM staging system. Figure 4 shows a clear risk stratification of survival times using nomogram-predicted survival probabilities. Therefore, using this nomogram, physicians can predict their patients' prognosis, provide more informative explanations to the patients, and initiate more individualised management.

There are limitations to be addressed in the present study. First, all potential predictive variables could never be included in the analysis to generate a nomogram with an absolute predictive ability. However, the internal validation demonstrated good fitness of the present nomogram based on the six variables for survival prediction. Nonetheless, we should recognise that bootstrapping is a sample reuse method that is useful to mitigate an overfit bias of the data for nomogram generation, but cannot ensure applicability to an external cohort. Therefore, our nomogram should be externally validated using an independent population in the future. Second, despite our use of a large multicenter collaborative database that provides prospectively collected data on patients with nonresectable pancreatic cancer, some of the patients were not followed-up until death. 
In conclusion, the present nomogram can predict the prognosis of patients with unresectable pancreatic cancer receiving gemcitabine-based chemotherapy with considerable accuracy, potentially facilitating highly tailored patient management.

\section{ACKNOWLEDGEMENTS}

We are indebted to Dr Yukihiro Koike, Kanto Central Hospital; Dr Ryou Nakata, Japanese Red Cross Medical Center; Dr Nobuo Toda, Mitsui Memorial Hospital; and Dr Makoto Okamoto, JR Tokyo General Hospital for their assistance in the data collection.

\section{CONFLICT OF INTEREST}

The authors declare no conflict of interest.

\section{REFERENCES}

Brennan MF, Kattan MW, Klimstra D, Conlon K (2004) Prognostic nomogram for patients undergoing resection for adenocarcinoma of the pancreas. Ann Surg 240(2): 293-298.

Brookmeyer R, Crowley J (1982) A confidence interval for the median survival time. Biometrics 38: 29-41.

Burris 3rd HA, Moore MJ, Andersen J, Green MR, Rothenberg ML, Modiano MR, Cripps MC, Portenoy RK, Storniolo AM, Tarassoff P, Nelson R, Dorr FA, Stephens CD, Von Hoff DD (1997) Improvements in survival and clinical benefit with gemcitabine as first-line therapy for patients with advanced pancreas cancer: a randomized trial. J Clin Oncol 15(6): 2403-2413.

Conroy T, Desseigne F, Ychou M, Bouche O, Guimbaud R, Becouarn Y, Adenis A, Raoul JL, Gourgou-Bourgade S, de la Fouchardiere C, Bennouna J, Bachet JB, Khemissa-Akouz F, Pere-Verge D, Delbaldo C, Assenat E, Chauffert B, Michel P, Montoto-Grillot C, Ducreux M. Groupe Tumeurs Digestives of U, Intergroup P (2011) FOLFIRINOX versus gemcitabine for metastatic pancreatic cancer. N Engl J Med 364(19): $1817-1825$.

Edge SB, Byrd DR, Compton CC, Fritz AG, Greene FL, Trotti A (2010) AJCC Cancer Staging Manual. (ed 7) Springer: New York.

Ferrone CR, Kattan MW, Tomlinson JS, Thayer SP, Brennan MF, Warshaw AL (2005) Validation of a postresection pancreatic adenocarcinoma nomogram for disease-specific survival. J Clin Oncol 23(30): 7529-7535.

Harrell FE Jr. RMS: Regression Modeling Strategies R Package version 3.4-0. http://CRAN.Rproject.org/package_rms.

Heinemann V, Quietzsch D, Gieseler F, Gonnermann M, Schonekas H, Rost A, Neuhaus H, Haag C, Clemens M, Heinrich B, Vehling-Kaiser U, Fuchs M, Fleckenstein D, Gesierich W, Uthgenannt D, Einsele H, Holstege A, Hinke A, Schalhorn A, Wilkowski R (2006) Randomized phase III trial of gemcitabine plus cisplatin compared with gemcitabine alone in advanced pancreatic cancer. J Clin Oncol 24(24): 3946-3952.

Iasonos A, Schrag D, Raj GV, Panageas KS (2008) How to build and interpret a nomogram for cancer prognosis. J Clin Oncol 26(8): 1364-1370.

Ikeda M, Okada S, Tokuuye K, Ueno H, Okusaka T (2001) Prognostic factors in patients with locally advanced pancreatic carcinoma receiving chemoradiotherapy. Cancer 91(3): 490-495.

International Bladder Cancer Nomogram ConsortiumBochner BH, Kattan MW, Vora KC (2006) Postoperative nomogram predicting risk of recurrence after radical cystectomy for bladder cancer. J Clin Oncol 24(24): 3967-3972.

Ishii H, Okada S, Nose H, Yoshimori M, Aoki K, Okusaka T (1996) Prognostic factors in patients with advanced pancreatic cancer treated with systemic chemotherapy. Pancreas 12(3): 267-271.

Kalbfleisch JD, Prentice RL (1980) The Statistical Analysis of Failure Time Data. Wiley: New York.

Kattan MW, Karpeh MS, Mazumdar M, Brennan MF (2003) Postoperative nomogram for disease-specific survival after an R0 resection for gastric carcinoma. J Clin Oncol 21(19): 3647-3650.

Kattan MW, Leung DH, Brennan MF (2002) Postoperative nomogram for 12-year sarcoma-specific death. J Clin Oncol 20(3): 791-796.
Klein LW, Block P, Brindis RG, McKay CR, McCallister BD, Wolk M, Weintraub W, Registry A-N (2002) Percutaneous coronary interventions in octogenarians in the American College of Cardiology-National Cardiovascular Data Registry: development of a nomogram predictive of in-hospital mortality. J Am Coll Cardiol 40(3): 394-402.

Lee C, He H, Jiang Y, Di Y, Yang F, Li J, Jin C, Fu D (2013) Elevated expression of tumor miR-222 in pancreatic cancer is associated with Ki67 and poor prognosis. Med Oncol 30(4): 700.

Liu R, Chen X, Du Y, Yao W, Shen L, Wang C, Hu Z, Zhuang R, Ning G, Zhang C, Yuan Y, Li Z, Zen K, Ba Y, Zhang CY (2012) Serum microRNA expression profile as a biomarker in the diagnosis and prognosis of pancreatic cancer. Clin Chem 58(3): 610-618.

Moore MJ, Goldstein D, Hamm J, Figer A, Hecht JR, Gallinger S, Au HJ, Murawa P, Walde D, Wolff RA, Campos D, Lim R, Ding K, Clark G, Voskoglou-Nomikos T, Ptasynski M, Parulekar W. National Cancer Institute of Canada Clinical Trials Group (2007) Erlotinib plus gemcitabine compared with gemcitabine alone in patients with advanced pancreatic cancer: a phase III trial of the National Cancer Institute of Canada Clinical Trials Group. J Clin Oncol 25(15): 1960-1966.

Nakai Y, Isayama H, Ijichi H, Sasaki T, Kogure H, Yagioka H, Miyabayashi K, Mizuno S, Yamamoto K, Mouri D, Kawakubo K, Yamamoto N, Hirano K, Sasahira N, Tateishi K, Tada M, Koike K (2012a) Phase I trial of gemcitabine and candesartan combination therapy in normotensive patients with advanced pancreatic cancer: GECA1. Cancer Sci 103(8): 1489-1492.

Nakai Y, Isayama H, Ijichi H, Sasaki T, Takahara N, Ito Y, Matsubara S, Uchino R, Yagioka H, Arizumi T, Hamada T, Miyabayashi K, Mizuno S, Yamamoto K, Kogure H, Yamamoto N, Hirano K, Sasahira N, Tateishi K, Tada M, Koike K (2013) A multicenter phase II trial of gemcitabine and candesartan combination therapy in patients with advanced pancreatic cancer: GECA2. Invest New Drugs 31(5): 1294-1299.

Nakai Y, Isayama H, Sasaki T, Sasahira N, Ito Y, Kogure H, Togawa O, Matsubara S, Arizumi T, Yagioka H, Yashima Y, Kawakubo K, Mizuno S, Yamamoto K, Hirano K, Tsujino T, Ijichi H, Tateishi K, Toda N, Tada M, Omata M, Koike K (2010) Impact of S-1 on the survival of patients with advanced pancreatic cancer. Pancreas 39(7): 989-993.

Nakai Y, Isayama H, Sasaki T, Sasahira N, Tsujino T, Kogure H, Yagioka H, Yashima Y, Togawa O, Arizumi T, Matsubara S, Hirano K, Tada M, Omata M, Koike K (2011) Comorbidity, not age, is prognostic in patients with advanced pancreatic cancer receiving gemcitabine-based chemotherapy. Crit Rev Oncol Hematol 78(3): 252-259.

Nakai Y, Isayama H, Sasaki T, Sasahira N, Tsujino T, Toda N, Kogure H, Matsubara S, Ito Y, Togawa O, Arizumi T, Hirano K, Tada M, Omata M, Koike K (2012b) A multicentre randomised phase II trial of gemcitabine alone vs gemcitabine and S-1 combination therapy in advanced pancreatic cancer: GEMSAP study. Br J Cancer 106(12): 1934-1939.

Nakai Y, Kawabe T, Isayama H, Sasaki T, Yagioka H, Yashima Y, Kogure H, Arizumi T, Togawa O, Ito Y, Matsubara S, Hirano K, Sasahira N, Tsujino T, Tada M, Omata M (2008) CA 19-9 response as an early indicator of the effectiveness of gemcitabine in patients with advanced pancreatic cancer. Oncology 75(1-2): 120-126.

Sakahara H, Endo K, Nakajima K, Nakashima T, Koizumi M, Ohta H, Hidaka A, Kohno S, Nakano Y, Naito A, Suzuki T, Torizuka K (1986) Serum CA 19-9 concentrations and computed tomography findings in patients with pancreatic carcinoma. Cancer 57(7): 1324-1326.

Sezgin C, Karabulut B, Uslu R, Sanli UA, Goksel G, Yuzer Y, Goker E (2005) Gemcitabine treatment in patients with inoperable locally advanced/ metastatic pancreatic cancer and prognostic factors. Scand J Gastroenterol 40(12): 1486-1492.

Siegel R, Naishadham D, Jemal A (2013) Cancer statistics, 2013. CA Cancer J Clin 63(1): 11-30.

Sugihara T, Yasunaga H, Horiguchi H, Fujimura T, Nishimatsu H, Kume H, Ohe K, Matsuda S, Fushimi K, Homma Y (2013a) Longer operative time is associated with higher risk of severe complications after percutaneous nephrolithotomy: Analysis of 1511 cases from a Japanese nationwide database. Int J Urol 20(12): 1193-1198.

Sugihara T, Yasunaga H, Horiguchi H, Nishimatsu H, Kume H, Ohe K, Matsuda S, Fushimi K, Homma Y (2013b) A nomogram predicting severe adverse events after ureteroscopic lithotripsy: 12372 patients in a Japanese national series. BJU Int 111(3): 459-466.

Sun C, Ansari D, Andersson R, Wu DQ (2012) Does gemcitabine-based combination therapy improve the prognosis of unresectable pancreatic cancer? World J Gastroenterol 18(35): 4944-4958. 
Therasse P, Arbuck SG, Eisenhauer EA, Wanders J, Kaplan RS, Rubinstein L, Verweij J, Van Glabbeke M, van Oosterom AT, Christian MC, Gwyther SG (2000) New guidelines to evaluate the response to treatment in solid tumors. European Organization for Research and Treatment of Cancer, National Cancer Institute of the United States, National Cancer Institute of Canada. J Natl Cancer Inst 92(3): 205-216.

Tian F, Appert HE, Myles J, Howard JM (1992) Prognostic value of serum CA 19-9 levels in pancreatic adenocarcinoma. Ann Surg 215(4): 350-355.

Touijer K, Scardino PT (2009) Nomograms for staging, prognosis, and predicting treatment outcomes. Cancer 115(13 Suppl): 3107-3111.

Ueno H, Ioka T, Ikeda M, Ohkawa S, Yanagimoto H, Boku N, Fukutomi A, Sugimori K, Baba H, Yamao K, Shimamura T, Sho M, Kitano M, Cheng AL, Mizumoto K, Chen JS, Furuse J, Funakoshi A, Hatori T, Yamaguchi T, Egawa S, Sato A, Ohashi Y, Okusaka T, Tanaka M (2013) Randomized phase III study of gemcitabine plus S-1, S-1 alone, or gemcitabine alone in patients with locally advanced and metastatic pancreatic cancer in Japan and Taiwan: GEST study. J Clin Oncol 31(13): 1640-1648.

Ueno H, Okada S, Okusaka T, Ikeda M (2000) Prognostic factors in patients with metastatic pancreatic adenocarcinoma receiving systemic chemotherapy. Oncology 59(4): 296-301.

Von Hoff DD, Ervin T, Arena FP, Chiorean EG, Infante J, Moore M, Seay T, Tjulandin SA, Ma WW, Saleh MN, Harris M, Reni M, Dowden S, Laheru D, Bahary N, Ramanathan RK, Tabernero J, Hidalgo M, Goldstein D, Van Cutsem E, Wei X, Iglesias J, Renschler MF (2013) Increased survival in pancreatic cancer with nab-paclitaxel plus gemcitabine. N Engl J Med 369(18): 1691-1703.

This work is published under the standard license to publish agreement. After 12 months the work will become freely available and the license terms will switch to a Creative Commons AttributionNonCommercial-Share Alike 3.0 Unported License. 